Table 1. Relative Risk of Gout by Quintiles of DASH and Western Diet Score, Stratified by Mean GRS

\begin{tabular}{|c|c|c|c|c|c|c|c|c|c|c|c|}
\hline & \multicolumn{11}{|c|}{ DASH } \\
\hline & \multicolumn{4}{|c|}{ Below Mean } & \multicolumn{7}{|c|}{ Above Mean } \\
\hline & Q1 & Q2 & Q3 & Q4 & Q5 & Q1 & Q2 & Q3 & Q4 & Q5 & P Interaction \\
\hline No. Cases & 27 & 49 & 51 & 21 & 22 & 75 & 89 & 90 & 34 & 65 & \\
\hline Person-Years & 39208 & 47247 & 57227 & 34953 & 58764 & 39815 & 45853 & 55401 & 34733 & 56521 & \\
\hline Age-Adjusted RR & 1.0 (ref) & $1.43(0.89,2.29)$ & $1.22(0.76,1.96)$ & $0.8(0.45,1.42)$ & $0.5(0.28,0.88)$ & 1.0 (ref) & $0.97(0.72,1.33)$ & $0.79(0.58,1.07)$ & $0.47(0.31,0.70)$ & $0.54(0.39,0.76)$ & 0.73 \\
\hline \multirow[t]{3}{*}{ MV-Adjusted ${ }^{*}$ RR } & $\begin{array}{r}1.0 \text { (ref) } \\
\text { Western }\end{array}$ & $1.56(0.97,2.51)$ & $1.32(0.82,2.12)$ & $0.89(0.50,1.59)$ & $0.61(0.34,1.09)$ & 1.0 (ref) & $1.0(0.73,1.37)$ & $0.85(0.63,1.17)$ & $0.51(0.33,0.76)$ & $0.68(0.49,0.96)$ & 0.69 \\
\hline & $\begin{array}{l}\text { Western } \\
\text { Below Mean }\end{array}$ & & & & & Above Mear & & & & & \\
\hline & Q1 & Q2 & Q3 & Q4 & Q5 & Q1 & Q2 & Q3 & Q4 & Q5 & P Interaction \\
\hline No. Cases & 21 & 36 & 28 & 39 & 46 & 52 & 70 & 56 & 76 & 99 & \\
\hline Person-Years & 47397 & 49348 & 47837 & 47589 & 45283 & 45529 & 47913 & 47357 & 46 & 44785 & \\
\hline Age-Adjusted RR & 1.0 (ref) & $1.49(0.86,2.56)$ & $1.26(0.71,2.23)$ & $1.71(1.00,2.93)$ & $2.22(1.31,3.74)$ & 1.0 (ref) & $1.21(0.85,1.74)$ & $0.98(0.67,1.43)$ & $1.35(0.94,1.93)$ & $1.88(1.34,2.65)$ & 0.72 \\
\hline MV-Adjusted ${ }^{*}$ RR & 1.0 (ref) & $1.34(0.78,2.32)$ & $1.07(0.60,1.90)$ & $1.33(0.76,2.34)$ & $1.63(0.91,2.93)$ & 1.0 (ref) & $1.17(0.81,1.68)$ & $0.93(0.63,1.38)$ & $1.27(0.87,1.84)$ & $1.77(1.19,2.61)$ & 0.64 \\
\hline
\end{tabular}

*Adjusted for age (continuous), menopause, use of hormone therapy (never, past or current), history of hypertension, systolic and diastolic blood pressure (continuous), alcohol (continuous), total energy intake (continuous), and intake of meat, seafood, and dairy foods (continuous).

genetically predisposed females was larger with greater absolute risk difference. These data agree with the recent GBD Study's recommendation for intensive dietary and anti-obesity measures for gout prevention, especially in females. ${ }^{1}$ REFERENCES:

[1] Xia et al., PMID 31624843

[2] Keller et al., PMID: 28487277

[3] Tin et al., PMID 31578528

[4] Wallace et al., PMID: 856219

Acknowledgements: The authors thank the participants of the NHS.

CY is supported by the Rheumatology Research Foundation Scientist Development Award and NIH T32 AR007258. HC is supported by NIH P50AR060772 and R01AR065944.

Disclosure of Interests: None declared

DOI: 10.1136/annrheumdis-2021-eular.3758

\section{OP0204 URATE CRYSTAL DEPOSITIONS ARE ASSOCIATED WITH INFLAMMATORY MARKERS AND CAROTID PLAQUES INDICATING SUBCLINICAL INFLAMMATION IN GOUT; BASELINE RESULTS FROM THE NOR-GOUT STUDY}

H. B. Hammer ${ }^{1,2}$, S. Rollefstad3, G. Jensen3, L. Karoliussen3, L. Terslev ${ }^{4}$, E. A. Haavardsholm ${ }^{2,3}$, T. K. Kvien ${ }^{2,3}$, A. G. Semb3, T. Uhlig ${ }^{2,3}$. 'Diakonhjemmet Hospital, Department of Rheumatology, Oslo, Norway; ${ }^{2}$ University of Oslo, Faculty of Medicine, Oslo, Norway; ${ }^{1}$ Diakonhjemmet Hospital, Department of Rheumatology, Oslo, Norway; ${ }^{4}$ Rigshospitalet Glostrup, Centre for Rheumatology and Spinal Diseases, Copenhagen, Denmark

Background: In gout patients the extent of monosodium urate (MSU) crystal depositions can be quantitated by use of ultrasound. Gout may be associated with atherosclerotic disease which might be related to low-grade inflammation. Calprotectin is a major granulocyte protein reflecting the level of systemic inflammation. Objectives: To explore whether the extent of MSU crystal depositions is associated with low grade systemic inflammation as assessed by calprotectin/C-reactive protein (CRP) and with carotid pathologies in asymptomatic gout patients. Methods: The baseline data from NOR-GOUT, a prospective study of patients with crystal-proven gout with increased serum urate levels $(>360 \mu \mathrm{mol} / \mathrm{L})$, were used. All patients were assessed by ultrasound (GE Logiq E9 machine) to assess MSU depositions (OMERACT definitions; double contour (DC), tophi and aggregates) with bilateral assessment of wrist, MCP2, knee, ankle, MTP1 and insertions of triceps, quadriceps, proximal/distal patellar and Achilles tendons, all scored semi-quantitatively 0 -3. Bilateral B-mode ultrasound assessments of the carotid arteries examined carotid intima-media thickness (cIMT; increased thickness $>0.9 \mathrm{~mm}$ ). Atherosclerotic plaques were identified as protrusions of $\geq 1.5 \mathrm{~mm}$ or $\geq 2$ times the adjacent IMT. Sum scores of each of the ultrasound elementary lesions for MSU depositions were calculated and the associations with calprotectin (plasma assessed by ELISA (Calpro), normal levels $<910 \mu \mathrm{g} / \mathrm{L}$ ), CRP (routine assessment, normal levels $<4 \mathrm{mg} / \mathrm{L}$ ) as well as cIMT and presence of carotid plaque were explored. Correlations were performed by use of Spearman and differences between group was investigated by Mann-Whitney test.

Results: A total of 202 gout patients without flare were included $(95.5 \%$ men, mean (SD) age of 56.5 (13.8) years, 7.9 (7.7) years since first flare). The mean (SD) sum sore of DC was $4.4(3.5)$, tophi $6.6(6.6)$, aggregates $9.3(5.6)$, calprotectin 801 (525) $\mu \mathrm{g} / \mathrm{L}$, CRP 7 (14) mg/L and serum urate (SUA) 499 (77) $\mu \mathrm{mol} / \mathrm{L}$. Carotid examinations were performed in $122(60.4 \%)$ of the patients. Significant correlations were found between the MSU depositions and the inflammatory markers as well as cIMT. The $27 \%$ patients with increased calprotectin had the highest levels of each of the three elementary lesions ( $p=0.001$ ) (Figure), and the $39 \%$ with elevated CRP levels had the highest levels of tophi and aggregates $(p<0.05)$. The $18 \%$ with increased thickness of cIMT and the $53 \%$ of patients with carotid plaque had the highest levels of aggregates $(p=0.003$ and $p=0.037$, respectively).
Conclusion: In asymptomatic gout patients, higher load of MSU crystal depositions was associated with increased levels of inflammatory markers, cIMT and presence of atherosclerotic plaques in the carotid arteries. This may indicate that crystal depositions cause subclinical inflammation with subsequent systemic implications, but future longitudinal studies are needed to confirm such causal relationships.

\begin{tabular}{lccc}
\hline & $\begin{array}{c}\text { Sum score } \\
\text { double contour }\end{array}$ & $\begin{array}{c}\text { Sum score } \\
\text { tophi }\end{array}$ & Sum score aggregates \\
\hline Calprotectin & $0.26^{* *}$ & $0.32^{\star *}$ & $0.28^{\star *}$ \\
CRP & $0.20^{*}$ & $0.25^{\star *}$ & $0.18^{*}$ \\
Right IMT & NS & NS & $0.18^{*}$ \\
Left IMT & NS & NS & $0.21^{*}$ \\
Sum bilateral IMT & NS & NS & $0.22^{*}$ \\
\hline
\end{tabular}

Spearman's correlations. NS; Not significant, ${ }^{*} p<0.05,{ }^{* *} p<0.001$

Disclosure of Interests: Hilde Berner Hammer Speakers bureau: AbbVie, Lilly and Novartis, Silvia Rollefstad: None declared, Gro Jensen: None declared, Lars Karoliussen: None declared, Lene Terslev Speakers bureau: Speakers fee from AbbVie, Janssen, Roche, Novartis, Pfizer, MSD, BMS and GE, Espen A Haavardsholm: None declared, Tore K. Kvien Speakers bureau: Tore K Kvien has received fees for speaking and/or consulting and/or research funding to Diakonhjemmet Hospital from AbbVie, Biogen, BMS, Celltrion, Egis, Evapharma, Ewopharma, Eli Lilly, Gilead, Hikma, MSD, Mylan, Novartis, Oktal, Pfizer, Sandoz, Sanofi and UCB., Anne Grete Semb: None declared, Till Uhlig: None declared

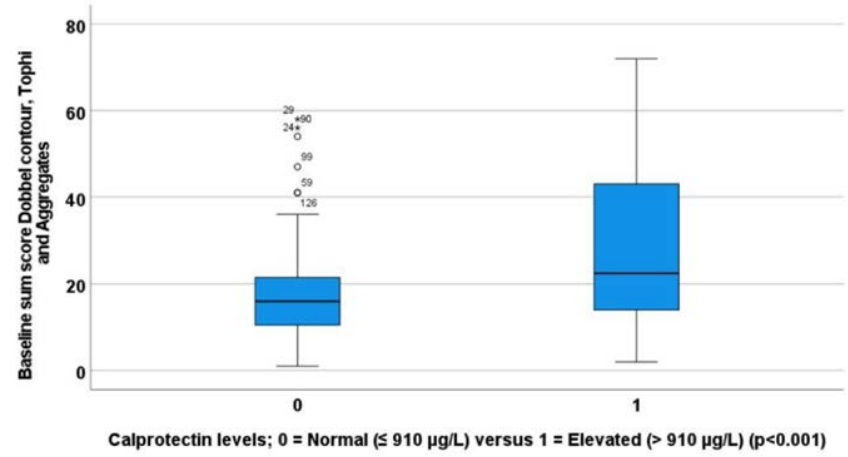

DOI: 10.1136/annrheumdis-2021-eular.290

\begin{tabular}{|l|l}
\hline OP0205 & ULTRASOUND-DETECTED CALCIUM \\
PYROPHOSPHATE CRYSTAL DEPOSITION: WHICH \\
SITES SHOULD BE SCANNED?
\end{tabular}

E. Cipolletta ${ }^{1}$, J. DI Battista ${ }^{1}$, W. Grassi ${ }^{1}$, E. Filippucci ${ }^{1}{ }^{1}$ Polytechnic University of Marche, Department of Clinical and Molecular Sciences, Ancona, Italy

Background: In recent years, ultrasonography (US) has emerged as an accurate and reliable tool for the diagnosis of calcium pyrophosphate (CPP) deposition disease (CPPD) in daily practice. Previous studies analyzed the diagnostic value of US findings in different tissues and joints. However, no studies have investigated the optimal US scanning protocol in the diagnosis of CPPD at patient level. Objectives: To assess the diagnostic value of the combinations of OMERACT-defined US findings of CPPD in the upper and lower limbs and to select the best minimal combination of anatomic structures to be scanned for diagnosing CPPD in inter-critical periods. 
Methods: Patients with a crystal-proven diagnosis of CPPD and age- and sexmatched disease-controls were prospectively enrolled in this cross-sectional, monocentric, case-control study. All subjects underwent a bilateral US examination of 9 hyaline cartilages $(\mathrm{HC}), 6$ fibrocartilages $(\mathrm{FC}), 5$ tendons, 1 joint recess and 1 ligament as follows: shoulder (glenoid $\mathrm{FC}$, humeral $\mathrm{HC}$ and acromioclavicular FC), elbow (humeral $\mathrm{HC}$ and triceps tendon), wrist (triangular FC, scapho-lunate ligament, volar recess of the radio-lunate joint), hand ( $\mathrm{HC}$ of the metacarpophalangeal joints from $2^{\text {nd }}$ to $5^{\text {th }}$ finger), hip (acetabular FC and femoral $\mathrm{HC}$ ), knee (femoral condyles' HC, meniscal FC, patellar and quadriceps tendons), ankle (talar HC, Achilles tendon and plantar fascia). US assessment was carried-out by a rheumatologist blinded to clinical data. CPP deposits were identified as presence/absence, according to the OMERACT definitions [1]. Results: Ninety-five patients were enrolled: 45 CPPD patients (age: $72 \pm 10.6$ years, disease duration: $5.6 \pm 7.8$ years, female/male ratio: 1.3 ) and 50 age- and sex-matched disease-controls (18 with rheumatoid arthritis, 13 with osteoarthritis, 10 with psoriatic arthritis and 9 with gout).

The FC of the medial and lateral meniscus were the most frequently involved targets of CPP deposits in cases ( $81.8 \%$ and $77.3 \%$ of patients, respectively), followed by the triangular $\mathrm{FC}$ of the wrist $(68.2 \%)$, the $\mathrm{HC}$ of the femoral condyles $(54.5 \%)$, the scapho-lunate ligament $(52.3 \%)$ and the acetabular FC $(50.0 \%)$ In all these anatomical targets, US findings indicative of CPP deposits were detected in a significantly higher percentage of cases than controls $(p<0.01)$. The US scanning protocols that showed the best balance between sensitivity and specificity, the most sensitive and the most specific were shown in Table 1.

Table 1. Diagnostic performances of different US scanning protocols

\begin{tabular}{lcccc}
\hline Anatomical targets & SE & SP & LH+ & LH- \\
\hline Knee meniscal FC and wrist triangular & 0.86 & 0.86 & 6.35 & 0.13 \\
FC & $(0.76-0.96)$ & $(0.73-0.94)$ & $(3.17-12.72)$ & $(0.06-0.30)$ \\
Knee meniscal FC, wrist triangular FC & 0.93 & 0.82 & 5.19 & 0.08 \\
$\quad$ and hip acetabular FC & $(0.82-0.99)$ & $(0.69-0.91)$ & $(2.85-9.42)$ & $(0.03-0.24)$ \\
Hip acetabular FC, knee femoral & 0.67 & 0.96 & 16.67 & 0.35 \\
condyles' HC and & $(0.51-0.80)$ & $(0.86-0.99)$ & $(4.22-65.82)$ & $(0.23-0.53)$
\end{tabular}

wrist scapho-lunate ligament

LH: likelihood ratio, SE: sensitivity, SP: specificity, US: ultrasonography.

In all US scanning protocols, each anatomical target was assessed bilaterally.

Figure 1 includes representative pictures showing CPP crystal deposits in different anatomical targets.

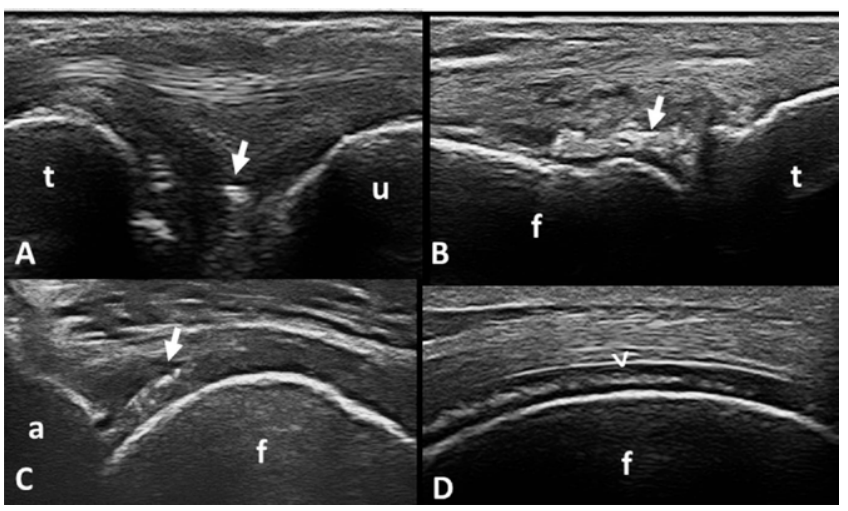

A: Wrist, longitudinal scan of the triangular FC complex.

$\mathrm{B}$ : Knee, longitudinal scan of the lateral meniscus.

C: Hip, longitudinal scan of the acetabular labrum FC,

D: Knee, longitudinal scan of the medial femoral condyle's HC.

Arrows: CPP crystal deposits at FC, arrowhead: CPP crystal deposits at $\mathrm{HC}$.

Conclusion: Our results suggest that bilateral US assessment of knee, wrist and hip provided acceptable sensitivity and specificity for diagnosing CPPD. REFERENCES:

[1] Filippou G, et al. Identification of calcium pyrophosphate deposition disease (CPPD) by ultrasound: reliability of the OMERACT definitions in an extended set of joints-an international multiobserver study by the OMERACT Calcium Pyrophosphate Deposition Disease Ultrasound Subtask Force. Ann Rheum Dis. 2018;77:1194-9.

Disclosure of Interests: Edoardo Cipolletta: None declared, Jacopo Di Battista: None declared, Walter Grassi Speakers bureau: Walter Grassi has received speaking fees from AbbVie, Celgene, Grünenthal, Pfizer and Union Chimique Belge Pharma., Emilio Filippucci Speakers bureau: Emilio Filippucci. has received speaking fees from AbbVie, Bristol-Myers Squibb, Janssen-Cilag, Novartis, Pfizer, Roche and Union Chimique Belge Pharma.

DOI: 10.1136/annrheumdis-2021-eular.496

\section{OP0206 \\ ASSOCIATION BETWEEN FEMALE REPRODUCTIVE FACTORS AND GOUT: NATIONWIDE POPULATION- BASED COHORT STUDY OF 1 MILLION POSTMENOPAUSAL WOMEN}

Y. Eun ${ }^{1}$, I.Y. Kim², K. D. $\mathrm{Han}^{3}$, K. Lee ${ }^{3}$, S. Y. Kang ${ }^{1}$, S. Lee ${ }^{1}$, H. S. Cha ${ }^{1}$, E. M. Koh' ${ }^{1}$, J. Lee ${ }^{1}$, H. Kim ${ }^{4} .{ }^{1}$ Samsung Medical Center, Sungkyunkwan University School of Medicine, Department of Medicine, Seoul, Korea, $c$ of (South Korea); ${ }^{2}$ National Police Hospital, Department of Medicine, Seoul, Korea, Republic of (South Korea); ${ }^{3}$ Soongsil University, Department of Statistics and Actuarial Science, Seoul, Korea, Republic of (South Korea); ${ }^{4}$ Samsung Medical Center, Sungkyunkwan University School of Medicine, Department of Medical Humanities, Seoul, Korea, Republic of (South Korea)

Background: Previous studies have shown that the incidence of gout differs according to gender, and its risk factors also differ according to gender. However little research has been done on the association between reproductive factors and gout.

Objectives: Our study attempted to determine whether there is an association between reproductive factors and the incidence of gout in a large nationwide population-based cohort of postmenopausal women.

Methods: Postmenopausal women aged 40-69 who participated in national health screenings in 2009 were included in the study. Subjects who had been diagnosed with gout prior to medical examination were excluded from the study, and a total of 1,076,378 women were included in the study. Outcome was the occurrence of gout which was defined as a case of two outpatient visit or one hospitalization for gout using the ICD-10 code of gout (M10) in the claim database. The Cox proportional hazard model was used for the analysis, and stratified analysis according to the body mass index (BMI) and chronic kidney disease (CKD) was performed.

Results: Mean follow-up duration was 8.1 years, and incident cases of gout were 64,052. Later menarche (adjusted HR 1.10, 95\% Cl 1.02-1.19 in >16 years compared with $\leq 12$ years), earlier menopause (adjusted HR 1.12 in $<40$ years, 1.06 in 40-45 years, 1.03 in 45-50 years, compared with 50-55 years), and shorter reproductive span (adjusted HR 1.10 in <30 years, 1.06 in 30-35 years, compared with $\geq 40$ years) were associated with a high risk of gout. No association between parity and gout incidence was observed. Use of oral contraceptives (OC; adjusted HR 1.03 in <1 year, $1.05 \geq 1$ year, compared with non-user) and hormone replacement therapy (HRT; adjusted HR 1.16 in <2 years, 1.16 in 2-5 years, 1.18 in $\geq 5$ years, compared with non-user) were associated with an increased risk of gout. The association between reproductive factors and gout remained in the trend without statistical significance in the low BMI group, but not in the high BMI group. The effects of use OC and HRT on gout were not significant in the CKD group.

Conclusion: Shorter exposure to endogenous estrogen was associated with a high risk of gout. Conversely, exposure to exogenous estrogen such as $\mathrm{OC}$ and HRT was associated with an increase in gout risk. This association was not significant in subjects with high BMI or CKD.

Disclosure of Interests: None declared

DOI: 10.1136/annrheumdis-2021-eular.4169

\section{OP0207 ASSOCIATION BETWEEN DEMENTIA AND GOUT: A NATIONWIDE POPULATION-BASED STUDY}

J. Kim ${ }^{1}$, H. M. P. Park ${ }^{2}$, D. H. Yim ${ }^{3}$, I. A. Choi ${ }^{4}$, S. Y. Eom ${ }^{5} .{ }^{1}$ Chungbuk National University Hospital, Internal Medicine, Division of Rheumatology, Cheongju-si, Chungcheongbuk-do, Korea, Republic of (South Korea); ${ }^{2}$ Chungbuk National University Hospital, Psychiatry, Cheongju, Korea, Republic of (South Korea); ${ }^{3}$ Chungbuk National University, Center for Environmental Medicine, Cheongju, Korea, Republic of (South Korea); ${ }^{4}$ Chungbuk National University College of Medicine, Division of Rheumatology, Department of Internal Medicine, Cheongju, Korea, Republic of (South Korea); ${ }^{5}$ College of Medicine, Chungbuk National University, Department of Preventive Medicine, Cheongju, Korea, Republic of (South Korea)

Background: Dementia is a common mental illness associated with aging Alzheimer's disease (AD) and Vascular dementia (VaD) are the most common causes of dementia in the elderly. In previous studies, hyperuricemia was suggested to have anti-oxidant effects and possible neuroprotective effects $[1,2]$.

Objectives: Hyperuricemia is the most important factor in the pathogenesis of gout. This study aims to investigate association between gout and dementia.

Methods: We conducted a nationwide population-based study using the NHISNSC database of the Korean populations consisted of 1 million individuals between 2002 and 2013. Gout patients were defined by International Classification of Diseases (ICD)-10 code for gout (M10) and prescriptions for urate lowering therapy including allopurinol, febuxostat and benzbromarone at least 30days. Dementia was also defined by ICD-10 codes for dementia; Alzheimer's disease (AD; F00), vascular dementia (VaD; F01). 